\title{
Time-Dependent Behavior of Insoluble Monomolecular Films: Fatty Acids and Some Derivatives
}

\author{
BERNARD SIMS ${ }^{1}$ AND GEORGE ZOGRAFI
}

\author{
University of Michigan, College of Pharmacy, Ann Arbor, Michigan 48104
}

Received April 7, 1971; accepted November 22, 1971

\begin{abstract}
Saturated fatty acids ranging from 15 to 20 carbons in alkyl chain length and some stearic acid derivatives were spread as insoluble monomolecular films and compressed at a constant velocity. As these surface films assume close packing, they exhibit a loss in surface pressure when held at constant area below an apparent collapse pressure which is not due to dissolution into the subphase solution. The higher the surface pressure to which the film is compressed, the greater the number of repeated compressions to a given surface pressure after periods of noncompression, and the longer the time period between successive compressions, the greater the rate and extent of instability. Increasing chain length increases film stability, but different behavior is noted for odd-and even-carbon acids in a manner analogous to their melting behavior. From these studies it is concluded that three-dimensional phase separation occurs when the film is compressed to surface pressures approaching the liquid condensed-solid condensed transition point. The relative rates of molecular expulsion from the surface, and of lens formation and growth determine the rate of surface pressure loss when compression is stopped. Similar studies with methyl stearate, 2-hydroxystearic acid, methyl 2-OH stearate and methyl $9-\mathrm{OH}$ stearate are consistent with these conclusions.
\end{abstract}

\section{INTRODUCTION}

Rabinovitch et al. (1) have shown that stearic acid monolayers spread on acidic solution lose surface pressure when held at constant area below the apparent collapse pressure. This has been reported for other fatty acids and fatty acid derivatives as well (2). What exactly gives rise to this pressure loss has not been defined clearly. However, previous studies have established that dissolution of these compounds into the acidic subphase does not occur (1-3). Rabinovitch et al. (1) suggested for stearic acid that at a critical area per molecule of about $21 \mathrm{~A}^{2}$ / molecule, molecules are ejected and that an "inversion" process takes place; molecules are pushed into the aqueous phase below the

1 Predoctoral training grant fellow GM 1367, National Institutes of Health, Bethesda, Md. Present address: The Upjohn Co., Kalamazoo, MI. monolayer or out above the monolayer to form a second layer. No further consideration was given toward establishing evidence for such behavior.

In recent studies from this laboratory the influence of compressional rate on the collapse behavior of fatty acid monolayers, ranging from pentadecanoic $\left(\mathrm{C}_{15}\right)$ to eicosanoic $\left(\mathrm{C}_{20}\right)$ acids, was reported (2). The apparent collapse pressure was shown to increase with increasing chain length, but the odd-carbon compounds always exhibited higher collapse pressures than the next higher even-carbon compound. Likewise, preliminary study indicated that the rates of surface pressure loss from initial values below the apparent collapse pressure were such that an odd compound appeared more stable than the next higher even-carbon compound (2).

Since it is known from melting point and thermal analysis data that an odd-carbon 35

Copyright (1) 1972 by Academio Press, Inc. All rights of reproduction in any form reserved. 
fatty acid crystal is in a higher state of energy than the next higher even-carbon compound, the previous results seem to imply that the process of molecular condensation to form lenses above the monolayer is the important kinetic factor in film stability. In other words, a shorter oddchain fatty acid might be ejected from the monolayer more readily than the next higher even-carbon compound, but the tendency of lens growth, being less for the odd-compound leads to a greater stability. Indeed the reverse process of spreading from fatty acid crystals has been shown to give spreading pressures corresponding to odd-even behavior, where odd-carbon compounds give higher equilibrium spreading pressures than the next higher even compound $(4,5)$.

In the present study we wished to consider this problem further and to see if surface pressure losses below collapse also are related to the formation of lenses and the rate of such lens formation. Our feelings in this regard were strengthened by some data presented by Gaines (13) for film instability of stearic acid at $24.8 \mathrm{dyn} / \mathrm{cm}$ surface pressure. Although this was below an apparent collapse surface pressure, he showed that the area required to maintain this surface pressure constant was reduced by $10 \%$ in 30 min. He further observed in this case the appearance of whitish streaks or collapsed monolayer.

\section{EXPERIMENTAL METHODS}

Materials. The fatty acids and esters used were all obtained from Applied Science Co. (State College, PA) except for methyl 9hydroxystearate which was the gift of Dr. A. Hoffman, Mayo Clinic, Rochester, MN. All substances were chromatographically pure.

Equipment. The monolayer balance utilized has been described previously (2). Surface pressure was measured by means of a modified Wilhelmy plate method using a roughened platinum plate. Mass changes were measured with a Cahn RG electrobalance and recorded on a Coleman-Hitachi 165 recorder. Since vertical movement with this balance is negligible the possibility of error in surface tension measurement due to contact angle change (6) is greatly mini- mized. This was confirmed previously by excellent agreement for values of the transition surface pressure for all fatty acid films, before and after collapse $(1,2)$.

Films were spread from hexane solution using a Burroughs-Wellcome Agla microliter syringe unit. The subphase for all fatty acids was $0.01 \mathrm{M} \mathrm{HCl}$, while that for the methyl esters was $0.01 M \mathrm{NaCl}$. The results were the same for the esters using $\mathrm{NaCl}$ or $\mathrm{HCl}$; but for studies where films were in contact with subphase for an extended period, it was felt that the possibility of acid hydrolysis should be avoided.

\section{RESULTS \\ Fatty Acids}

In all experiments, unless otherwise stated, a rate of compression of $1.2 \mathrm{~A}^{2} /$ molecule/ min was used. Similar behavior was noted for other compressional rates, but this rate was chosen because it gave a convenient range of surface pressures before collapse, as well as a suitable time period for obtaining a complete $\pi-A$ curve. All curves presented are taken from a specific recorder tracing but each represents two or three experiments with agreement within $0.5 \mathrm{dyn} /$ cm surface pressure.

Figure 1 represents an experiment with stearic acid designed to show that at this compressional rate: (i) instability sets in near the transition pressure; (ii) a sequence of compression and noncompression leads to greater surface pressure loss on standing; and (iii) a markedly reduced collapse pressure $^{2}$ now occurs at about $22.0 \mathrm{dyn} / \mathrm{cm}$ (last compression). This latter value can be contrasted with one of $49.8 \mathrm{dyn} / \mathrm{cm}$ which is the collapse pressure for stearic acid compressed directly to collapse at the same velocity.

Figure 2 indicates that even at values where films are apparently stable when first compressed, if subsequent conditions bring about an opportunity for instability to begin (viz compression to higher values of surface pressure but below collapse) even the lower pressure is now a region of instability. In

${ }^{2}$ Collapse pressure is the maximum surface pressure attained with continual compression at a constant rate. 


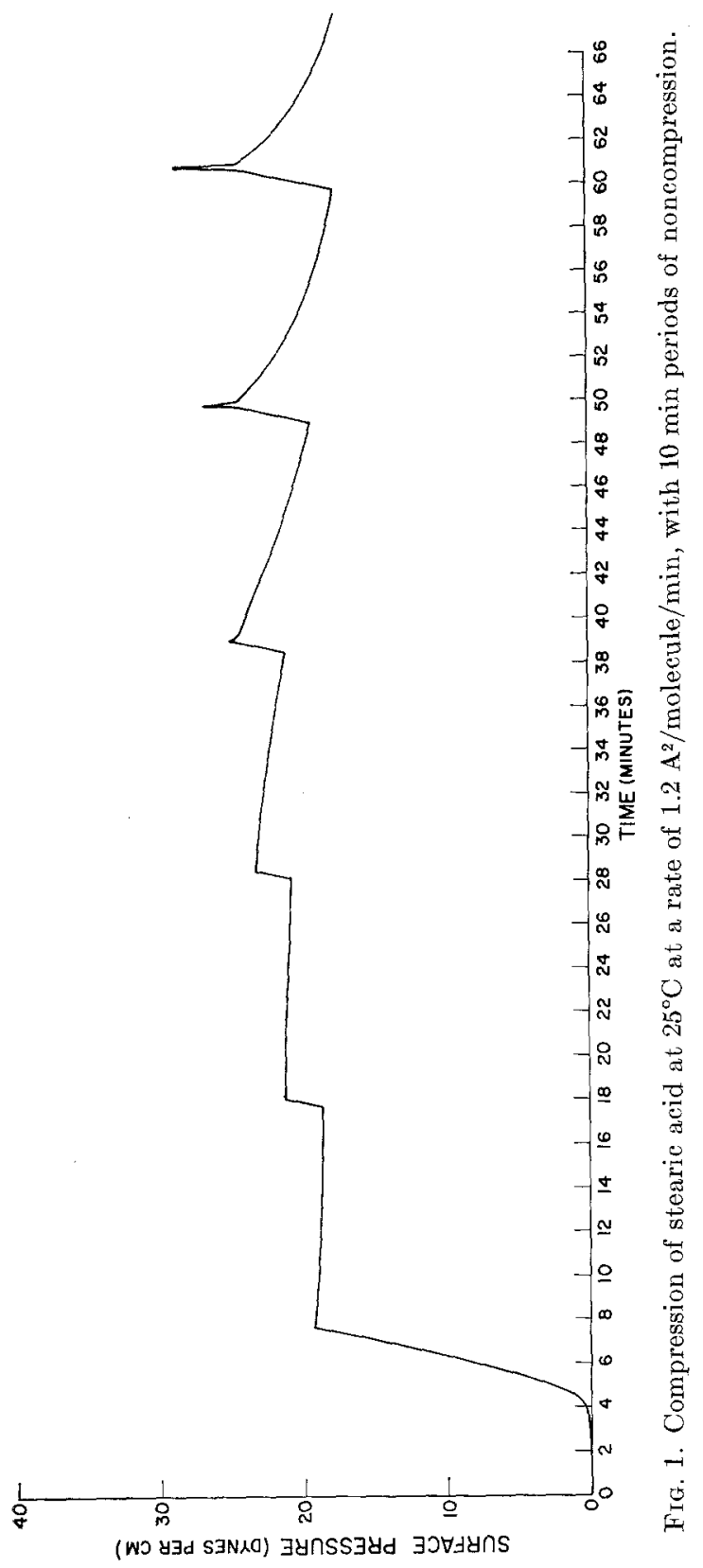




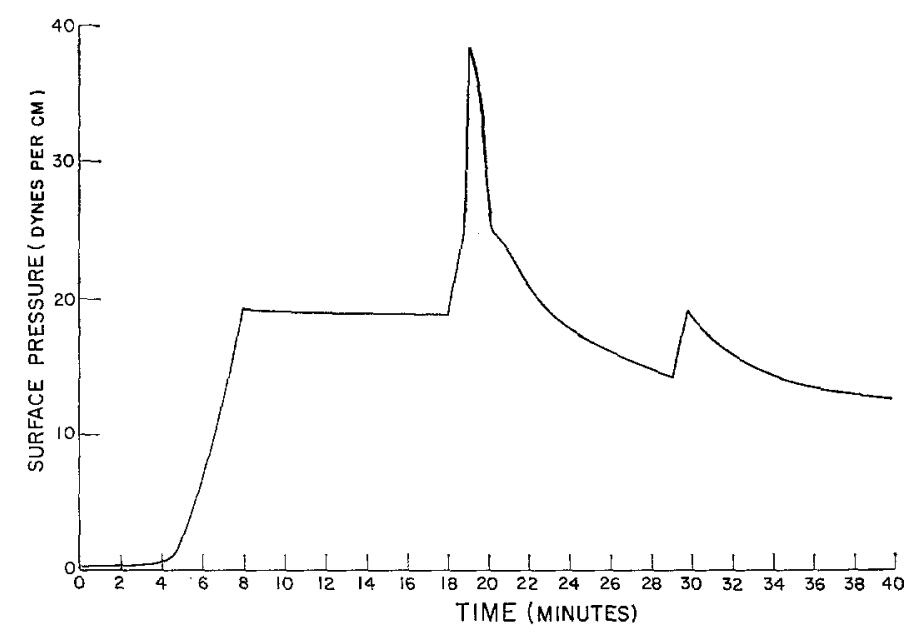

Fia. 2. Compression of stearic acid at $25^{\circ} \mathrm{C}$ at a rate of $1.2 \mathrm{~A}^{2} / \mathrm{molecule} / \mathrm{min}$ to $19.0,38.0$, and 19.0 dyn/em surface pressure with $10 \mathrm{~min}$ periods of noncompression between each compression step.

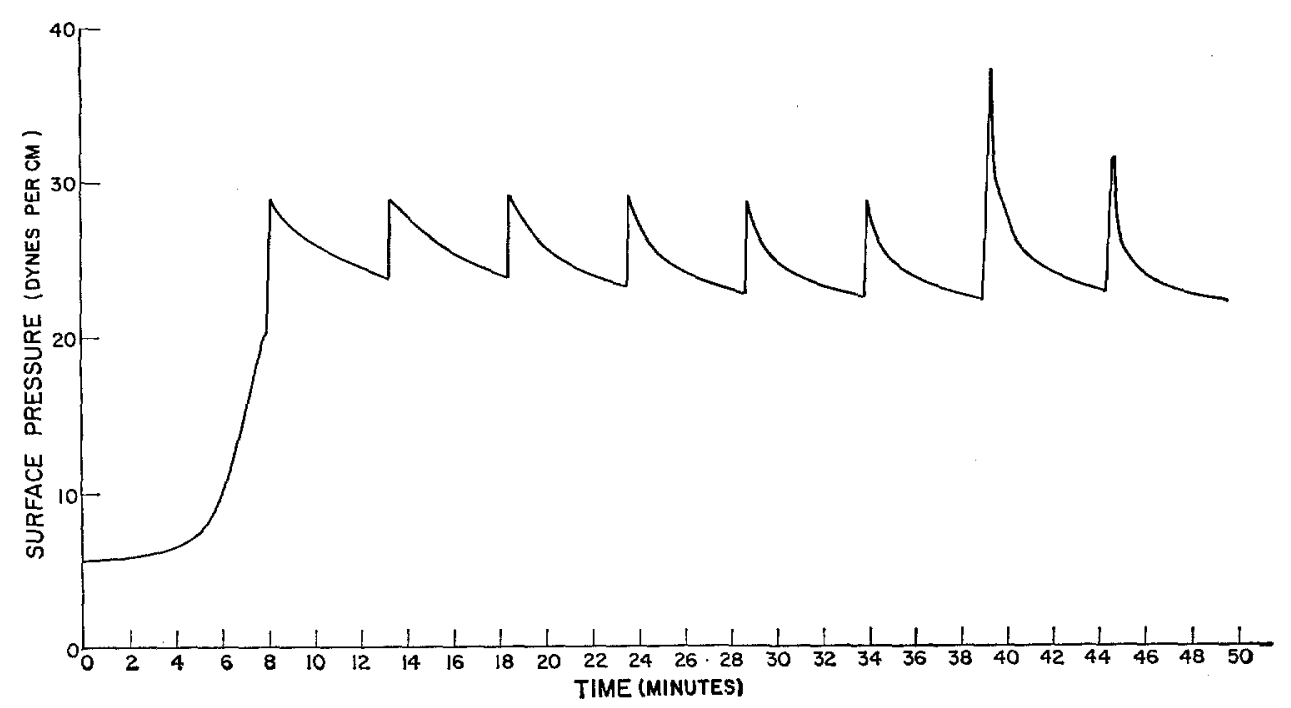

FIG. 3. Compression of pentadecanoic acid with 5 min periods of noncompression for 6 time periods at $28.5 \mathrm{dyn} / \mathrm{cm}$ followed by compression to collapse for 2 time periods at a rate of $1.2 \mathrm{~A}^{2} / \mathrm{molecule} / \mathrm{min}$.

general, the longer one allows the surface pressure fall to occur and the higher one compresses the film before stopping the compression the greater the instability observed upon further compression. ${ }^{3}$

To compare the relative stability for oddand even-carbon compounds the following experiments were performed. Each compound was compressed to a value below its

${ }^{3}$ Space does not permit the large number of plots showing this, but the interested reader may consult Ref. (7) for this data. apparent collapse pressure, held at constant area for $5 \mathrm{~min}$ and recompressed. This process was repeated a number of times. Figure 3 depicts the compression of pentadecanoic acid to $28.5 \mathrm{dyn} / \mathrm{cm}$, holding the area constant for $5 \mathrm{~min}$ and repeating the sequence 6 times. The monolayer was then compressed to an apparent collapse point and held at constant area before compressing again to a new apparent collapse point. The first collapse pressure is very close to the value of $35.3 \mathrm{dyn} / \mathrm{cm}$ observed earlier (2) for 


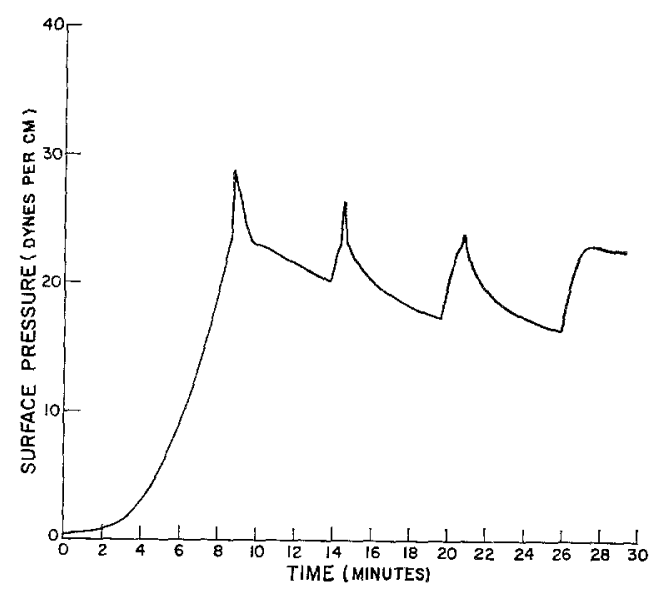

FIG. 4. Compression of palmitic acid with 5 min periods of noncompression for 3 time periods, after initial compression of the film to $38.0 \mathrm{dyn} / \mathrm{cm}$ at a rate of $1.2 \mathrm{~A}^{2} /$ molecule/min.

direct compression to collapse at $1.2 \mathrm{~A}^{2}$ / molecule/min, whereas the second value is reduced somewhat. ${ }^{4}$ A similar sequence was attempted with palmitic acid (see Fig. 4), however, after the first cycle a collapse point was obtained at $27.0 \mathrm{dyn} / \mathrm{cm}$ which is lower than that observed upon direct compression $(32.0 \mathrm{dyn} / \mathrm{cm})$. Thus, the shorter chain oddcarbon compound is seen to be much more stable upon repeated compression steps.

Similar comparisons were made with heptadecanoic, stearic, nonadecanoic, and eicosanoic acids compressed to $38.0 \mathrm{dyn} / \mathrm{cm}$, (below collapse) held at constant area for 5 min, and recompressed to $38.0 \mathrm{dyn} / \mathrm{cm}$ a number of times.

Figure 5 shows that noticeable collapse of heptadecanoic acid sets in during the sixth compression step, at about $29.0 \mathrm{dyn} / \mathrm{cm}$ (collapse point upon direct compression is $50.4 \mathrm{dyn} / \mathrm{cm}$ ). It is interesting to note the fourth and fifth compression steps since at about $32.0 \mathrm{dyn} / \mathrm{cm}$ an inflection oceurs which appears related to the collapse point. Also, each step produces an increasing rate of pressure loss. Again, as shown by comparison of pentadecanoic and palmitic acids, if heptadecanoic acid is compared with stearic acid (see Fig. 6) the odd-carbon compound

\footnotetext{
${ }^{4}$ Note in the first 6 compression sequences an increasing rate of surface pressure loss with each compression.
}

is much more stable. For one further comparison of odd- and even-carbon behavior, note the properties of nonadecanoic and eicosanoic acids in Figs. 7 and 8, where the longer chain compound is again much less stable. It should be stressed, however, that in all cases, increased compression-noncompression sequences lead to some increased instability.

As another indication of film stability, in a second series of experiments the surface pressure loss from collapse or below collapse after one compression step was followed for $12-18 \mathrm{hr}$ until a fairly constant value was reached. This has been termed the monolayer stability limit by Gaines (8) because it represents the apparent equilibrium surface pressure between a collapsed film and its monolayer. Note in Table I the alternation of values for odd- and even-carbon compounds, with the surface pressure of the lower-number odd chain exhibiting a higher monolayer stability limit. This is considered further in the Discussion section, but it should be noted that all time-dependent behavior observed at collapse or below collapse show this odd-even effect, which appears related to an equilibrium between crystalline forms and the monolayer, as shown by Cary and Rideal (4). Indeed, in a number of cases, it was possible to see lens formation with appropriate lighting.

Fatty Acid Derivatives (polar group effects)

In a previous study (2), the collapse pressures for some 2 -hydroxy fatty acids and the methyl esters of some normal fatty acids were compared with their corresponding normal fatty acids. In general the order of collapse pressures was 2-hydroxy fatty acid $>$ fatty acid $>$ methyl ester, except for one case where methyl palmitate had a higher collapse pressure than palmitic acid (at $1.2 \mathrm{~A}^{2} / \mathrm{molecule} / \mathrm{min}$ rate of compression). To study the influence of chemical modification on film stability and to relate this to the previous experiments with oddeven carbon compounds the following experiments were performed.

Figures 9 and 10 indicate the behavior of methyl stearate and 2-OH stearic acid when compressed in steps wih periods of noncompression. As with stearic acid, (in Fig. 1) 


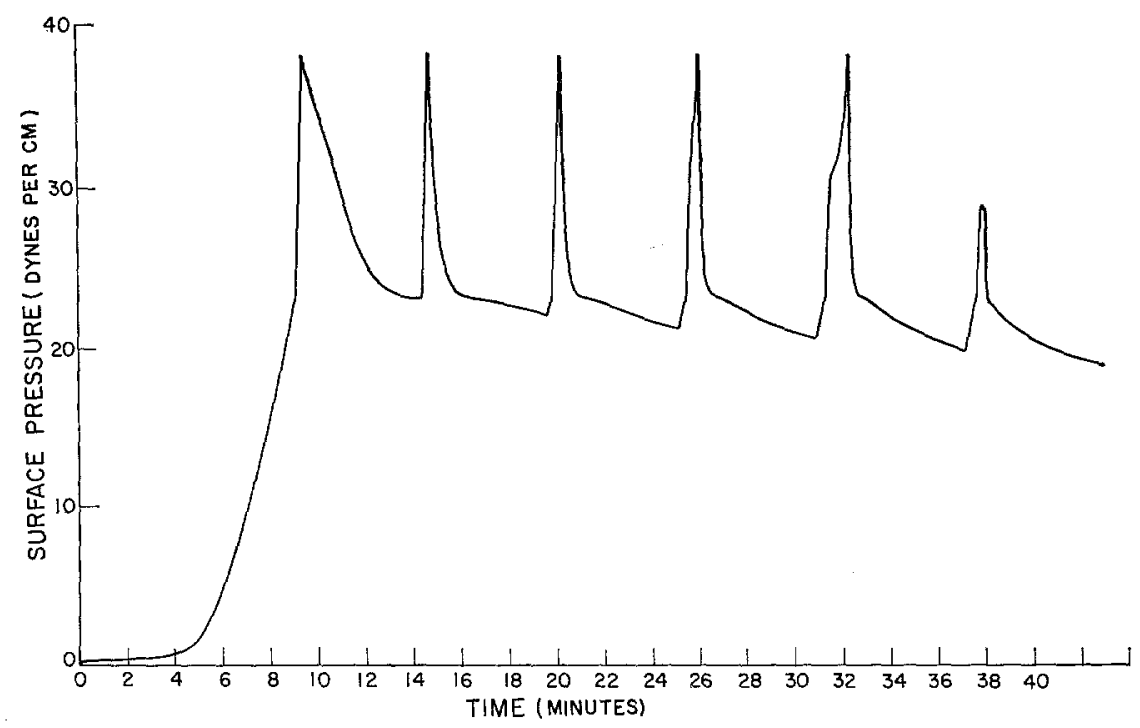

FIG. 5. Compression of heptadecanoic acid with 5 min periods of noncompression for 6 time periods, after initial compression of the film to $38.0 \mathrm{dyn} / \mathrm{cm}$ at a rate of $1.2 \mathrm{~A}^{2} / \mathrm{molecule} / \mathrm{min}$.

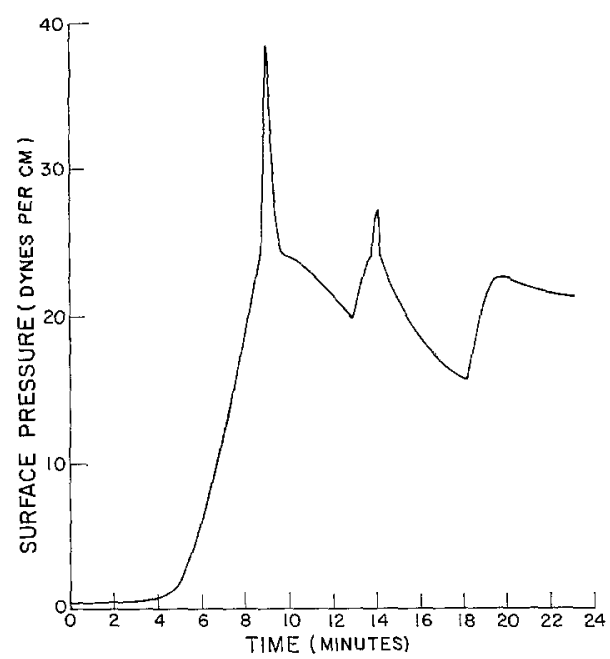

Fig. 6. Compression of stearic acid with $5 \mathrm{~min}$ periods of noncompression for 3 time periods after initial compression of the film to $38.0 \mathrm{dyn} / \mathrm{cm}$ at a rate of $1.2 \Lambda^{2} /$ molecule $/ \mathrm{min}$.

increasing the surface pressure of methyl stearate above its transition pressure (9.5 $\mathrm{dyn} / \mathrm{cm})$ greatly enhances the loss of surface pressure; at collapse, a surface pressure of $36.3 \mathrm{dyn} / \mathrm{cm}$ is obtained. This value can be compared to the value of $40.5 \mathrm{dyn} / \mathrm{cm}$ obtained for direct compression to collapse (1), however, this is a much smaller difference than the values of about 22.0 and $49.8 \mathrm{dyn} /$ $\mathrm{cm}$ obtained for stearic acid after stepwise and direct compression to collapse, respectively. On the other hand, Fig. 10 reveals the significant stability of $2-\mathrm{OH}$ stearic acid films right up to the collapse point and the very small difference noted between stepwise compression and direct compression to collapse. Another point of comparison is the effect of a continued number of compression steps after the first collapse pressure is reached. With stearic acid, the collapse pressure was reduced significantly with increasing number of compression steps, while Figs. 10 and 11 reveal that both methyl stearate and 2-OH stearic acid exhibit very stable collapse pressures upon such treatment, particularly the latter compound. If the monolayer is held at constant area after collapse, however, the surface pressure for methyl stearate slowly drops to values near its transition pressure while the 2-OH stearic acid film remains at significantly high pressures as shown in Fig. 12.

The collapse and pressure loss of the methyl ester of $2-\mathrm{OH}$ stearic acid was followed in order to compare with the compounds mentioned above. The compression at $1.2 \mathrm{~A}^{2} /$ molecule/min to collapse, followed 

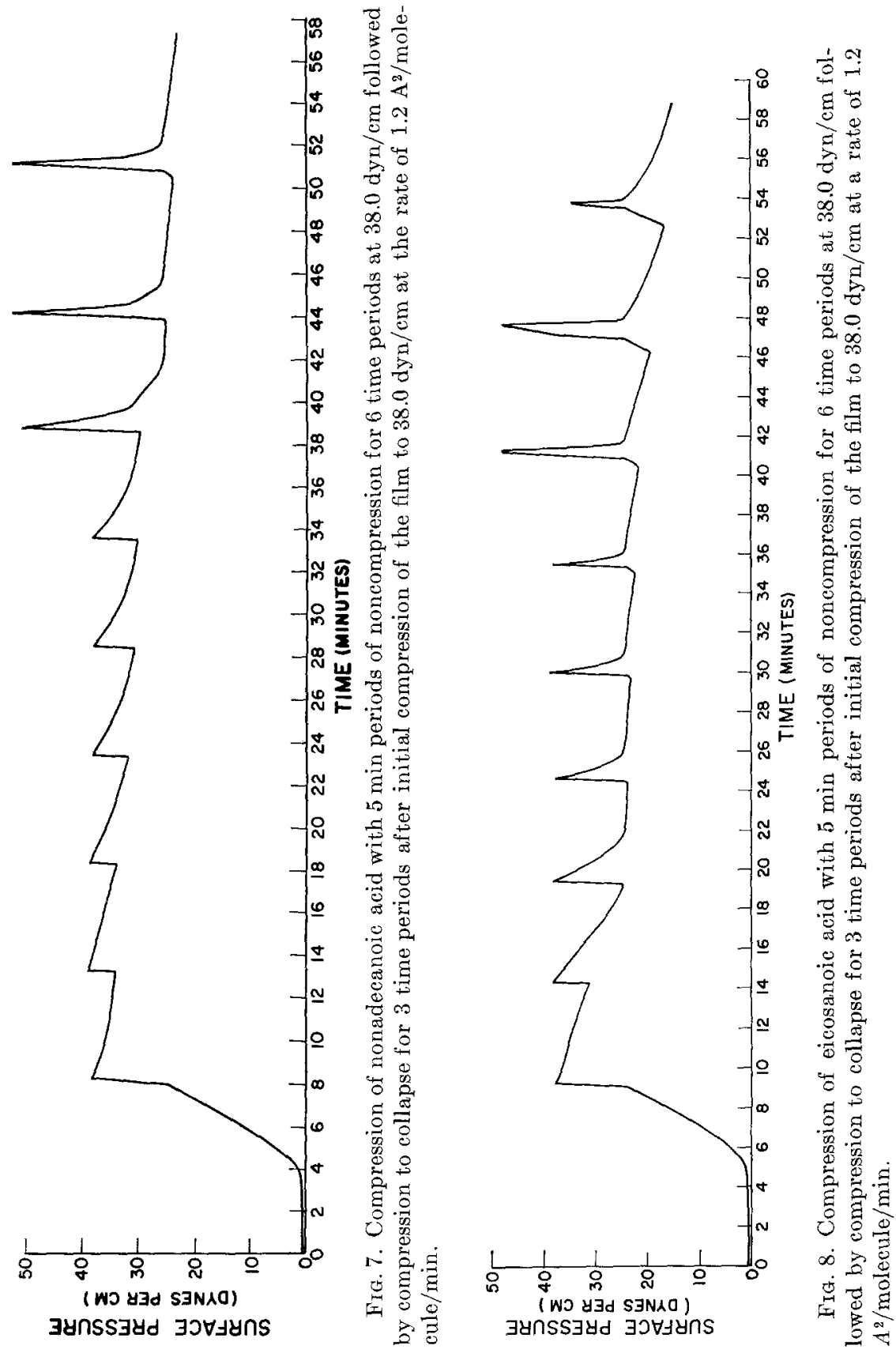
TABLE I

Comparison of apparent Equilibrium Pressures Between Monolayer and Lens FOR VARIOUS n-Saturated FatTy ACID Compounds

Compound $\quad \begin{gathered}\text { Monolayer stability limit } \\ (\text { dyn } / \mathrm{cm}) \pi_{\mathrm{eq}}\end{gathered}$

Pentadecanoic acid

10.7

Palmitic acid

9.8

Heptadecanoic acid

Stearic acid

15.2

Nonadeanoic acid $\quad 12.9$

Eicosanoic acid by a long period at constant area, gave a collapse pressure of $41.8 \mathrm{dyn} / \mathrm{cm}$ and exhibited only a $1.0 \mathrm{dyn} / \mathrm{cm}$ change over a period of $10 \mathrm{~min}$. Comparison of this compound with its $9-\mathrm{OH}$ isomer was carried out by compression from areas of $44 \mathrm{~A}^{2} /$ molecule to. $20 \mathrm{~A}^{2} /$ molecule, as shown in Fig. 13 . The 9-hydroxy derivative maintained constant surface pressure upon compression at about $12.0 \mathrm{dyn} / \mathrm{cm}$ between 44 and $30 \mathrm{~A}^{2} /$ molecule and then showed a rapid rise in pressure. At $38.0 \mathrm{dyn} / \mathrm{cm}$ compression was stopped, and a relatively rapid drop in surface pressure occurred (about $13.0 \mathrm{dyn} / \mathrm{cm}$ in $10 \mathrm{~min}$ ). Compression-expansion and recompression

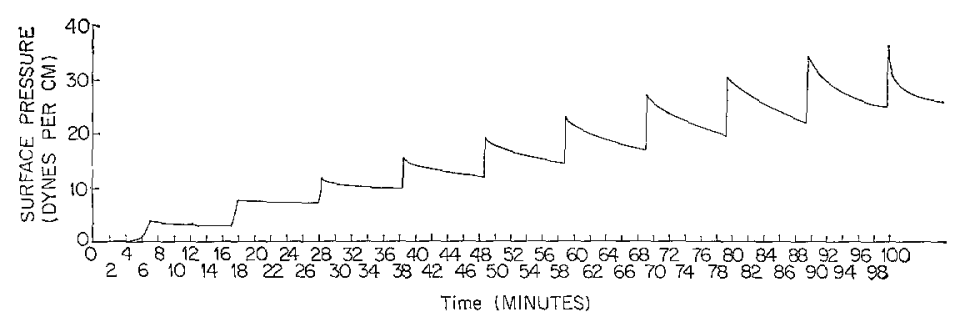

Frg. 9. Compression of methyl stearate on $0.01 \mathrm{M} \mathrm{NaCl}$ at $25^{\circ} \mathrm{C}$ at a rate of $1.2 \mathrm{~A}^{2} / \mathrm{molecule} / \mathrm{min}$, with 10 min periods of noncompression. Area per molecule at zero time is $30.0 \mathrm{~A}^{2}$.

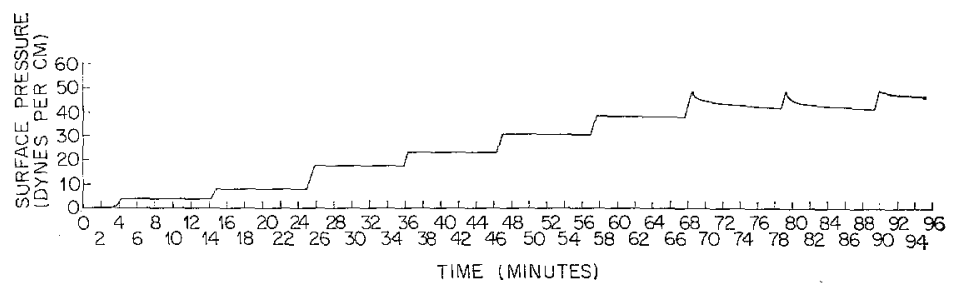

FIG. 10. Compression of 2-hydroxystearic acid at $25^{\circ} \mathrm{C}$ at a rate of $1.2 \mathrm{~A}^{2} / \mathrm{molecule} / \mathrm{min}$, with $10 \mathrm{~min}$ periods of noncompression. Area per molecule at zero time is $30.0 \mathrm{~A}^{2}$.

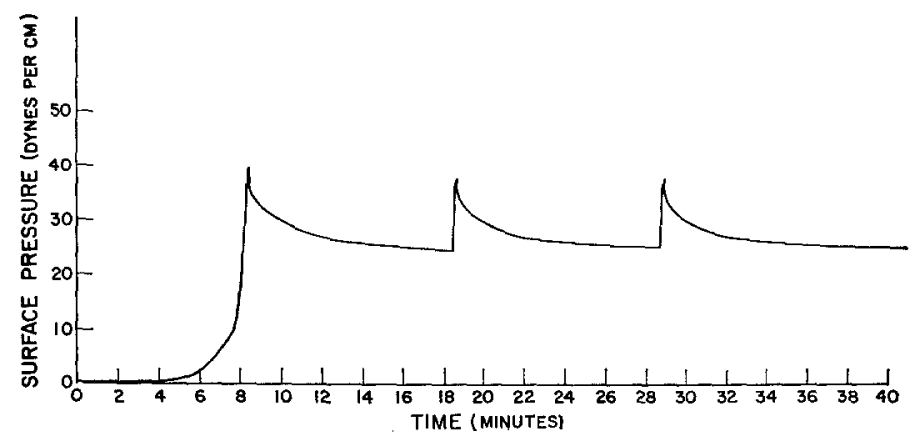

Fra. 11. Compression of methyl stearate with $10 \mathrm{~min}$ periods of noncompression for 3 time periods, after initial compression of the film to collapse at a rate of $1.2 \mathrm{~A}^{2} / \mathrm{molecule} / \mathrm{min}$. 


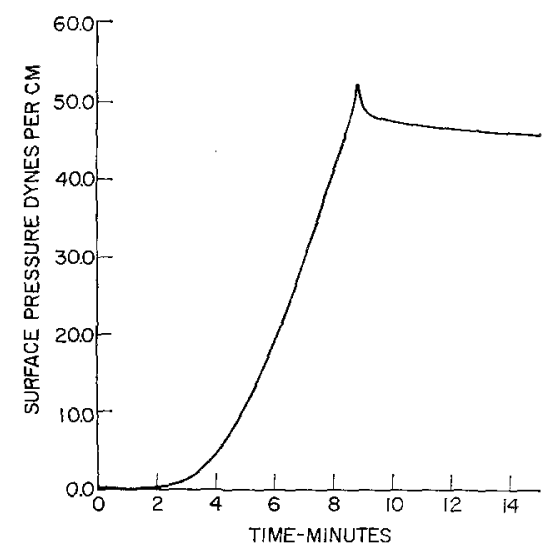

FIG. 12. Compression of 2-hydroxystearic acid at $25^{\circ} \mathrm{C}$ to collapse followed by a period of noncompression, at a rate of $1.2 \mathrm{~A}^{2} / \mathrm{molecule} / \mathrm{min}$. Area per molecule at zero time is $30.0 \mathrm{~A}^{2}$.

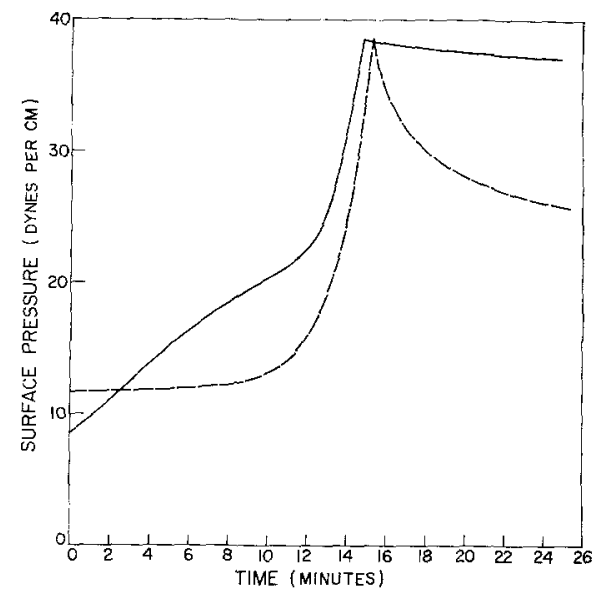

FIG. 13. Compression of 2-hydroxymethylstearate (-); and 9-hydroxymethylstearate (---) on $0.01 M \mathrm{NaCl}$ at $25^{\circ} \mathrm{C}$ to $38.0 \mathrm{dyn} / \mathrm{cm}$ surface pressure followed by a $10 \mathrm{~min}$ period of noncompression, at a rate of $1.6 \mathrm{~A}^{2} / \mathrm{molecule} / \mathrm{min}$ Area per molecule at zero time is $44.0 \mathrm{~A}^{2}$.

revealed identical curves, so dissolution was not responsible for this surface pressure loss. Different behavior was noted for the $2-\mathrm{OH}$ derivative which exhibited a pressure change from 8.4 to $19.0 \mathrm{dyn} / \mathrm{cm}$ in the range of 44 to $30 \mathrm{~A}^{2}$, followed by only a $1.0 \mathrm{dyn} / \mathrm{cm}$ fall in pressure from $38.0 \mathrm{dyn} / \mathrm{cm}$. Thus the change in position of the $\mathrm{OH}$ group significantly alters monolayer stability for this derivative.

\section{DISCUSSION}

In order to describe the mechanism(s) associated with instability of fatty acid monolayers it is useful to first consider the processes which give rise to surface pressure increase upon compression, and then to consider factors which can oppose these effects and lead to surface pressure loss.

It is generally considered that long chain fatty acids do not produce very much surface pressure above $30 \mathrm{~A}^{2} /$ molecule because of low surface coverage due to cohesion into groups of "islands" (9). At about $25 \mathrm{~A}^{2} /$ molecule, where surface pressure begins to change significantly, the surface coverage is increased as the islands are compressed to form a more continuous layer. During compression from the point of initial surface pressure development to the transition point, film molecules must assume various arrangements which allow for increased surface coverage and increased surface pressure. This can occur while the polar groups are still in one plane, followed by various degrees of staggering with vertical displacement into and out of the aqueous phase. The significant change in slope of the $\pi-A$ curve at the transition pressure must reflect a major shift in organization which in turn leads to an increased tendency above this point for film molecules to stagger. The significant increase in surface pressure produced by the increased surface coverage eventually will be balanced by an increasing tendency for ejection of molecules and a maximum "collapse" pressure will be attained. Exactly at what point specific arrangements occur is not clear, but since the major instability sets in as one passes the transition pressure, we can conclude that the barrier to measurable instability is overcome as the film molecules begin to rearrange to the more closely packed forms occurring around the transition pressure.

The results of these experiments suggest that, during compression, ejection of molecules leads to the formation of lenses and it is this which gives rise to time-dependent behavior. It is felt that the tendency for lens formation to occur actually begins at the monolayer stability limit, but that the speed of compression allows one to compress films 
to relatively high values of surface pressure. Factors favoring the formation and growth of lenses, therefore, should give rise to increased instability at low compression rates.

As noted earlier $(1,2)$ a reduced rate of compression significantly reduces the collapse pressure presumably because of more time for lens formation relative to the rate of increasing surface pressure. Even at lower pressures (below collapse), one should be able to induce the formation of lenses by repeated compression and with extended periods of noncompression between each compression step. Presumably each previous compression could provide sufficient nuclei to set off the process of lens formation, while longer periods in between could allow for condensation of molecules to form more and larger nuclei. This is supported by the data given previously in Figs. 1 and 2 and Ref. seven.

The appearance of three-dimensional lenses when films are compressed to the transition pressure and higher is supported by the observations made when comparing odd-and even-carbon fatty acids. As noted earlier (2) for any velocity, compression of the fatty acid monolayers to the transition pressure gives a regular increasing increment in transition pressure with increasing alkyl chain length. In contrast, the surface pressures observed for collapse alternate in an odd-even sequence, an odd-carbon fatty acid always giving a higher colapse pressure than the next higher even-carbon fatty acid. The first observation can be explained by assuming an increasing energy requirement to displace molecules into the configurations assumed at the transition pressure, the greater the number of $-\mathrm{CH}_{2}-$ groups the greater the energy requirement. Since ejection is just a continuation of this process, increasing chain length should favor a greater collapse pressure $\pi_{c}$. However, the process of condensation to form lenses also increases with chain length and this should reduce $\pi_{c}$. This latter factor would explain the alternation of $\pi_{c}$ for even- and odd-carbon fatty acids. Further, even-carbon fatty acid crystals ordinarily exhibit higher melting points than the next higher odd-carbon fatty acid, indicating that the even-carbon crystal is in a lower state of energy (10). In an analogous manner, therefore, the lens in contact with the even-carbon fatty acid monolayer should be in a lower state of energy with an increased tendency to accept monolayer molecules relative to the next higher odd-carbon compound. Therefore, despite an increase in chain length, which would normally favor a higher collapse pressure because of less ejection, one obtains a lower $\pi_{c}$ because of enhanced condensation when the increase is from an odd- to an even-carbon compound. Likewise if the presence of three-dimensional crystalline lenses is responsible for film instability before an apparent collapse point is reached, the final value of $\pi$, attained after an extended period of noncompression, which reflects an apparent equilibrium between this phase and the monolayer should show this odd and even behavior. The odd-even alternation noted in Table $I$ and the close correlation of these values with true equilibrium spreading pressures (ESP) for palmitic and stearic acids $(4,11,12)$ indicate that this is so. The value for pentadecanoic acid $(10.7 \mathrm{dyn} / \mathrm{cm})$ is much lower than the ESP of $21.0 \mathrm{dyn} / \mathrm{cm}$ reported by Boyd (12), but perhaps some dissolution in $0.01 \mathrm{M} \mathrm{HCl}$ occurs for this compound over the $18 \mathrm{hr}$ period. It should be stressed that the nature of the collapsed film lens need not be exactly the same as the crystal; however, these odd and even effects do support retention of the crystalline properties responsible for the behavior seen with pure crystals.

The key to monolayer instability is the loss of surface pressure at any pressure during compression. If, indeed, the presence of lenses on the monolayer surface and subsequent condensation are responsible for observed reductions in surface pressure, then in view of the effects of lenses on the collapse and the apparent equilibrium spreading pressures we also might expect different rates of pressure reduction for odd- and evencarbon compounds. For example, odd-carbon compounds should show slower rates of pressure loss and, hence, a greater capability toward repeated compression without significant loss of stability. As shown in Figs. $3-8$, for repeated cycling of monolayers of pentadecanoic through eicosanoic acids, such is the case. Each odd-carbon compound gives a slower rate of pressure loss from a 
given $\pi$ than the next higher even-carbon compound. In addition, the odd-carbon compound can be subjected to more periods of repeated compression before the onset of collapse. Another significant influence of condensation behavior on monolayers is dramatically shown by comparing nonadecanoic and eicosanoic acids, as depicted in Figs. 7 and 8, respectively. That both the odd-and even-carbon acids initially give low rates of condensation can be attributed to their long chain length, since cohesive interactions between alkyl chains of the film constitute a barrier to the ejection of molecules during compression. Upon repeated cycling, however, the eicosanoic acid monolayer progressively shows significantly increased rates of pressure loss relative to nonadecanoic acid because its ability to nucleate into lenses is greater. On compression of eicosanoic acid to its maximum pressure, high rates of condensation eventually lead to collapse of the monolayer at a surface pressure below that obtained by direct compression. Nonadecanoic acid also exhibits a high rate of pressure loss during periods of noncompression at its maximum pressure; however, the monolayer is capable of repeatedly developing this same maximum pressure upon recompression without being offset by the condensation rate. Hence, the odd-carbon compound, having a relatively lower rate of condensation, is significantly more stable than the next higher even-carbon compound.

With fatty acid derivatives, as with fatty acids, instability begins in the vicinity of the transition pressure, as shown in Fig. 9. The lower transition pressure and the greater instability at low pressures, for the methyl esters is probably caused by decreased polar interactions with water relative to the fatty acids and hence greater ease of ejection. An additional role of interaction between film molecules may be seen from the significant increase in the transition pressure and stability of the 2-OH fatty acids. The added $\mathrm{OH}$ group at the 2-position could be involved in intermolecular interactions with closely aligned polar groups, as well as with the underlying water molecules. It is clear that despite an apparent increased tendency for ejection, methyl ester molecules do not condense as rapidly as normal fatty acids to form lenses. This is seen by the small changes in collapse pressure when methyl stearate is repeatedly compressed back to collapse, as well as the small change in collapse pressure when the compressional velocity is reduced significantly. We would expect the extent of ejection to be the same regardless of compressional velocity, but a longer period should allow more time for lens formation and growth. Apparently this is why both palmitic acid at $1.2 \mathrm{~A}^{2} /$ molecule $/ \mathrm{min}$ (2) and stearic acid at $0.06 \mathrm{~A}^{2} /$ molecule $/ \mathrm{min}$ exhibit lower collapse pressures than their respective methyl esters. In view of the much lower melting point of each methyl ester relative to its corresponding fatty acid, it is not surprising that they would crystallize more slowly. Indeed for methyl stearate the fall in surface pressure from high initial values back to its transition pressure is much slower than that observed for stearic acid.

One might expect the $2-\mathrm{OH}$ acids to exhibit a greater tendency to form lenses since they have an additional polar group capable of hydrogen bonding; the melting points of these compounds are higher than their corresponding acids. On the other hand, a critical nucleus size must be reached to produce rapid lens growth. The repeated return to exactly the same collapse pressure after a small loss upon standing indicates no further lens growth probably because the monolayer resists further ejection beyond the point of closest packing. Such resistance to ejection is likely due to polar interactions between the film molecules, i.e., between carboxyl and hydroxyl groups.

Results with the 2-OH methyl stearate film are interesting because the collapse pressure is reduced to almost that of methyl stearate, yet upon standing there is minimal loss in pressure. as with the $2-\mathrm{OH}$ acid. Thus ejection is easier to bring about, but further loss of molecules to form larger lenses still does not appear to be energetically favorable. The replacement of the carboxyl proton by a methyl group should decrease interaction between film molecules and increase ejection ability while decreasing the tendency to form lenses.

The significant role played by the $2-\mathrm{OH}$ group in stabilizing fatty acid monolayers could best be evaluated by gradually altering 
the $\mathrm{OH}$-group position along the alkyl chain. The availability of the methyl ester of 9-OH stearic acid offers some clues since the behavior of the two isomer is quite different (Fig. 13). Of particular significance is the initial constancy of surface pressure upon compression of the 9-OH derivative up to $30 \mathrm{~A}^{2} /$ molecule, followed by a rapid rise. These results can be explained by assuming a horizontal orientation because of hydroxyl group interaction with water, as well as the greater surface coverage at these higher areas per molecule. However, as compression is continued the molecules are forced into an unfavorable vertical orientation. The rapid loss in surface pressure when compression is stopped (not due to dissolution) suggests that lens formation occurs readily since strong interactions with adjacent film molecules and with the subphase are not possible when the molecule is in a vertical orientation. Further work with isomers having hydroxyl groups at the 3 , and 4 positions would be most revealing in this regard.

\section{SUMMARY}

Saturated fatty acids with 15 to 20 carbons and some fatty acid derivatives were spread as insoluble monomolecular films, compressed at constant velocity, and their time-dependent behavior was studied. Comparison of the highest surface pressure reached before instability sets in goes in the order : 2 -OH fatty acids $>$ fatty acids $>$ methyl esters. Collapse pressures are generally greatest for the 2-OH fatty acids and lowest for the methyl esters, however, at lower compressional velocities methyl esters can exbibit higher collapse pressures than their corresponding fatty acids.

Fatty acids exhibit surface pressure loss when compressed above their transition pressure, but below an apparent collapse point, and held at constant area. Further, odd- and even-carbon. fatty acids exhibit alternating behavior with respect to collapse pressure, monolayer stability limit, and the rate of surface pressure loss at constant area; each odd-carbon compound exhibits greater stability than the next higher numbered even-carbon compound. Since this type of behavior is consistent with the melting eharacteristics and hence the crystallinity of fatty acids, it is concluded that the major factor contributing to the kinetics of loss in surface pressure upon standing is the formation of lenses above the monolayer, even before an apparent collapse point is reached.

\section{ACKNOWLEDGMEN'T}

This study was supported financially by the Merck, Sharp and Dohme Research Laboratories, West Point, PA.

\section{REFERENCES}

1. Rabinovitch, W., Robertson, R. F., AND Mason, S. G., Can. J. Chem. 38, 1881 (1960).

2. Sims, B., And Zograft, G., Chem. Phys. Lipids 6, 174 (1971).

3. Heikktla, R. E., Deamer, D. W. and CornWeLt, J. Lipid Res. 11, 195 (1970).

4. Cart, A., and Rtdeal, E. K., Proc. Roy. Soc., Ser. A 109, 318 (1925).

5. Adam, N. K., "The Physics and Chemistry of Surfaces," Dover ed., p. 104. Dover, New York, 1968.

6. Gaines, G. L., "Insoluble Monolayers at Liquid-Gas Interfaces," p. 347. Wiley (Interscience), New York, 1966.

7. Sims, B., "Dynamic Properties of Fatty Acids," $\mathrm{PhD}$ thesis, Univ. of Michigan, Ann Arbor, 1970.

8. Ref. (6), p. 45.

9. Davies, J., and Ridwal, E. K., "Interfacial Phenomena," 2nd ed., p. 239. Academic Press, New York, 1963.

10. Bailey, A., "Melting and Solidification of Fats," p. 146, Table 17. Wiley (Interscience), New York 1950.

11. Heikrila, R. E., Kwong, C. N., and CornWell, D. G., J. Lipid Res. 11, 190 (1970).

12. Boyd, G., J. Phys. Chem. 62, 536 (1958).

13. Ref. (6), p. 144. 